ANNALES

POLONICI MATHEMATICI

$96.2(2009)$

\title{
Pluriharmonic extension in proper image domains
}

\author{
by RAFAe CzYŻ (Kraków)
}

\begin{abstract}
Let $D_{j}$ be a bounded hyperconvex domain in $\mathbb{C}^{n_{j}}$ and set $D=D_{1} \times \cdots \times$ $D_{s}, j=1, \ldots, s, s \geq 3$. Also let $\Omega_{\pi}$ be the image of $D$ under the proper holomorphic map $\pi$. We characterize those continuous functions $f: \partial \Omega_{\pi} \rightarrow \mathbb{R}$ that can be extended to a real-valued pluriharmonic function in $\Omega_{\pi}$.
\end{abstract}

1. Introduction. For each $j=1, \ldots, s, s \geq 3$, let $D_{j}$ be a bounded hyperconvex domain in $\mathbb{C}^{n_{j}}, n_{j} \geq 1$. Recall that a bounded domain $\Omega \subseteq \mathbb{C}^{n}$, is called hyperconvex if there exists a plurisubharmonic function $\varphi: \Omega \rightarrow$ $(-\infty, 0)$ such that the closure of the set $\{z \in \Omega: \varphi(z)<c\}$ is compact in $\Omega$, for every $c \in(-\infty, 0)$. A bounded hyperconvex domain $\Omega$ in $\mathbb{C}^{n}$, viewed as a domain in $\mathbb{R}^{2 n}$, is always regular with respect to the Dirichlet problem for the Laplace operator (see e.g. [4]). Set

$$
D=D_{1} \times \cdots \times D_{s}, \quad j=1, \ldots, s, s \geq 3 .
$$

Then $D \subseteq \mathbb{C}^{n}, n=n_{1}+\cdots+n_{s}$, is a hyperconvex domain (see e.g. [2, Proposition 2.1]). Let $U$ be an open neighborhood of $\bar{D}$ and $\pi: U \rightarrow \mathbb{C}^{n}$, $n=n_{1}+\cdots+n_{s}$, be a proper holomorphic map. Set $\Omega_{\pi}=\pi(D)$. Then $\pi(\partial D)=\partial \Omega_{\pi}$, since $\pi$ is a proper map (see e.g. [6]). Furthermore, $\Omega_{\pi}$ is hyperconvex (Proposition 2.1).

Let $f: \partial \Omega_{\pi} \rightarrow \mathbb{R}, s \geq 3$, be a continuous function. Our main goal in Section 2 is to characterize those continuous functions $f$ that can be extended to real-valued pluriharmonic functions in $\Omega_{\pi}$. We prove:

Theorem A. Let $D_{j}$ be a bounded hyperconvex domain in $\mathbb{C}^{n_{j}}, n_{j} \geq 1$. Set $D=D_{1} \times \cdots \times D_{s}, j=1, \ldots, s, s \geq 3$. Moreover, let $U$ be an open neighborhood of $\bar{D}$, let $\pi: U \rightarrow \mathbb{C}^{n}, n=n_{1}+\cdots+n_{s}$, be a proper holomorphic map and let $\Omega_{\pi}=\pi(D)$. If $f: \partial D \rightarrow \mathbb{R}, n \geq 3$, is a continuous function, then the following assertions are equivalent:

2000 Mathematics Subject Classification: Primary 31C10; Secondary 32U15.

Key words and phrases: analytic discs, Dirichlet problem, Jensen measures, pluriharmonic functions, proper maps. 
(1) there exists a function $h$ that is pluriharmonic on $\Omega_{\pi}$, continuous on $\bar{\Omega}_{\pi}$ and $\left.h\right|_{\partial \Omega_{\pi}}=f$,

(2) $f$ is pluriharmonic on $\partial \Omega_{\pi}$ in the sense of Definition 2.2 ,

(3) the Perron-Bremermann envelope $\mathrm{PB}_{f}$ is pluriharmonic on $\Omega_{\pi}$, i.e.,

$$
\mathrm{PB}_{-f}=-\mathrm{PB}_{f},
$$

(see Section 2 for the definition of the Perron-Bremermann envelope),

(4) for every $z_{0} \in \partial \Omega_{\pi}$ and every Jensen measure $\mu \in \mathcal{J}_{z_{0}}^{c}$ with barycenter $z_{0}$ we have

$$
f\left(z_{0}\right)=\int_{\partial \Omega_{\pi}} f d \mu .
$$

Furthermore, if we assume that $\Omega_{\pi}$ has the approximation property, then the above conditions are equivalent to:

(5) the function $\mathrm{PB}_{-f}+\mathrm{PB}_{f}$ has smallest maximal plurisubharmonic majorant identically zero,

(6) $\lim \sup _{\Omega \ni z \rightarrow \xi}\left(\mathrm{PB}_{f}+\mathrm{PB}_{-f}\right)(z)=0$ for every $\xi \in \partial \Omega_{\pi}$.

(see Section 2 for the definition of the approximation property).

An elementary example of a continuous function $f: \partial \Omega_{\pi} \rightarrow \mathbb{R}$ satisfying the above conditions is the following: Let $u$ be holomorphic in a neighborhood of $\bar{\Omega}_{\pi}$ and let $f=\operatorname{Re}(u)$ be defined on $\partial \Omega_{\pi}$. Then $h=\operatorname{Re}(u)$ satisfies (1).

Let $s=n_{1}=1$. Then property (2) in Theorem A does not make sense and properties (1), (3)-(6) are true for every continuous function $f: \Omega_{\pi} \rightarrow \mathbb{R}$. If $s=2$, then it is in general not true that (2) implies (1) ([2, Example 3.4]), and if $s \geq 3$ and $\pi$ is the identity map or is such that $\Omega_{\pi}$ is the symmetrized polydisc (see e.g. [5] for the definition), then Theorem A was obtained in [2]. The equivalence between (1) and (3) was proved for an arbitrary hyperconvex domain in [1].

This article is organized as follows. The equivalence between assertions (1)-(4) is proved in Section 3 and the final part is proved in Section 4. In Section 5 we study plurisubharmonic boundary values in terms of analytic discs, in the case when $D=D_{1} \times \cdots \times D_{n}$ is a hyperconvex domain in $\mathbb{C}^{n}$.

2. Definitions, basic facts and notations. Let $D_{j}$ be a bounded hyperconvex domain in $\mathbb{C}^{n_{j}}, n_{j} \geq 1$, and set

$$
D=D_{1} \times \cdots \times D_{s} \subseteq \mathbb{C}^{n},
$$

where $n=n_{1}+\cdots+n_{s}$. For an open neighborhood $U$ of $\bar{D}$ and a proper holomorphic map $\pi: U \rightarrow \mathbb{C}^{n}$ we use the notation $\Omega_{\pi}=\pi(D)$. 
Let $I_{k}=\left(j_{1}, \ldots, j_{k}\right)$ be an increasing multi-index of length $k: 1 \leq j_{1}<$ $\cdots<j_{k} \leq s$, where $1 \leq k \leq s$. Define

$$
\Lambda^{I_{k}}=D_{1} \times \cdots \times \overbrace{\partial D_{j_{1}}}^{j_{1}} \times \cdots \times \overbrace{\partial D_{j_{k}}}^{j_{k}} \times \cdots \times D_{s} \quad \text { and } \quad \Lambda_{\pi}^{I_{k}}=\pi\left(\Lambda^{I_{k}}\right) .
$$

Hence,

$$
\partial D=\bigcup_{I_{k}} \Lambda^{I_{k}} \quad \text { and } \quad \partial \Omega_{\pi}=\pi(\partial D)=\bigcup_{I_{k}} \Lambda_{\pi}^{I_{k}} .
$$

Finally, denote by $\partial D^{+}$the distinguished boundary of $D$, i.e.

$$
\partial D^{+}=\partial D_{1} \times \cdots \times \partial D_{s} .
$$

Proposition 2.1. The domain $\Omega_{\pi}$ is hyperconvex.

Proof. For every $j$, let $\varphi_{j}$ be an exhaustion function for $D_{j}$. Then

$$
u\left(\zeta_{1}, \ldots, \zeta_{s}\right)=\max \left\{\varphi_{1}\left(\zeta_{1}\right), \ldots, \varphi_{s}\left(\zeta_{s}\right)\right\}
$$

is a plurisubharmonic exhaustion function for $D$ in $\mathbb{C}^{n}$. Now define

$$
\varphi(w)=\max \left\{u(z): z \in \pi^{-1}(w)\right\} .
$$

From [7] it follows that $\varphi$ is a plurisubharmonic exhaustion function for $\Omega_{\pi}$. Thus, $\Omega_{\pi}$ is hyperconvex.

Definition 2.2. An upper semicontinuous function $u: \partial \Omega_{\pi} \rightarrow \mathbb{R} \cup$ $\{-\infty\}$ is plurisubharmonic if $u$ is plurisubharmonic on every $\Lambda_{\pi}^{I_{k}}$ for every increasing multi-index $I_{k}=\left(j_{1}, \ldots, j_{k}\right)$ of length $k<s$, i.e., for every $w_{j_{1}} \in$ $\partial D_{j_{1}}, \ldots, w_{j_{k}} \in \partial D_{j_{k}}$, the function defined by

$$
u_{w_{j_{1}}}, \ldots, w_{j_{k}}:\left(z_{1}, \ldots, z_{s-k}\right) \mapsto u \circ \pi\left(z_{1}, \ldots, w_{j_{1}}, \ldots, w_{j_{k}}, \ldots, z_{s-k}\right)
$$

is plurisubharmonic on

$$
D_{I_{k}}=D_{1} \times \cdots \times \widehat{\partial D_{j_{1}}} \times \cdots \times \widehat{\partial D_{j_{k}}} \times \cdots \times D_{s} .
$$

The identically $-\infty$ function is by fiat not considered as plurisubharmonic. In a similar manner a continuous function $u: \partial \Omega_{\pi} \rightarrow \mathbb{R}$ is pluriharmonic if $u$ is pluriharmonic on every $\Lambda_{\pi}^{I_{k}}$ for every increasing multi-index $I_{k}$ of length $k<s$.

REMARK. Note that, if we take $\pi=\mathrm{id}_{D}$ in Definition 2.2, then an upper semicontinuous function $u$ is plurisubharmonic on $\partial D$ if for every increasing multi-index $I_{k}$ the restriction of $u$ to $\Lambda^{I_{k}}$ is plurisubharmonic.

The following definition comes from [11].

Definition 2.3. Let $\Omega \subseteq \mathbb{C}^{n}$. We say that $\Omega$ has the approximation property if for all upper bounded plurisubharmonic functions $u$ in $\Omega$ there exists a decreasing sequence $u_{j} \in \mathcal{P} \mathcal{S H}(\Omega) \cap C(\bar{\Omega})$ such that $u_{j} \rightarrow u^{*}$ on $\bar{\Omega}$. 
Wikström proved that B-regular domains and polydiscs have the approximation property (see [11]).

Definition 2.4. Let $\Omega \subseteq \mathbb{C}^{n}$ be a bounded domain and let $\mu$ be a nonnegative, regular Borel measure on $\bar{\Omega}$. The measure $\mu$ is a Jensen measure with barycenter at $z \in \bar{\Omega}$ for continuous plurisubharmonic functions if

$$
u(z) \leq \int_{\bar{\Omega}} u d \mu
$$

for every continuous function $u \in \mathcal{P} \mathcal{S H}(\Omega)$. The set of all such measures will be denoted by $\mathcal{J}_{z}^{c}$.

Similarly the measure $\mu$ is a Jensen measure with barycenter at $z \in \bar{\Omega}$ for upper bounded plurisubharmonic functions if

$$
u^{*}(z) \leq \int_{\bar{\Omega}} u^{*} d \mu
$$

for every upper bounded function $u \in \mathcal{P S H}(\Omega)$. The set of all such measures will be denoted by $\mathcal{J}_{z}$.

It is clear that $\left\{\delta_{z}\right\} \subset \mathcal{J}_{z} \subset \mathcal{J}_{z}^{c}$, where $\delta_{z}$ denotes the Dirac measure at $z$. If $\Omega$ is a hyperconvex domain, then $\operatorname{supp} \mu \subset \partial \Omega$ for all $z \in \partial \Omega$ and all $\mu \in \mathcal{J}_{z}^{c}$ (see [3]). Moreover, for a bounded hyperconvex domain $\Omega \subseteq \mathbb{C}^{n}$ it was proved in [11] that $\Omega$ has the approximation property if, and only if, $\mathcal{J}_{z}^{c}=\mathcal{J}_{z}$ for all $z \in \bar{\Omega}$.

We say that there exists a strong plurisubharmonic barrier at $z \in \partial \Omega$ if there exists $u \in \mathcal{P S H}(\Omega) \cap C(\bar{\Omega})$ such that $u(z)=0$ and $u<0$ in $\bar{\Omega} \backslash\{z\}$. A bounded domain $\Omega$ in $\mathbb{C}^{n}$ is called B-regular (see [9]) if for each $z \in \partial \Omega$ there exists a strong plurisubharmonic barrier at $z$.

Proposition 2.5. Let $\Omega \subset \mathbb{C}^{n}$ be bounded domain and let $z \in \partial \Omega$ be such that there exists a strong plurisubharmonic barrier at z. Then $\mathcal{J}_{z}^{c}=\left\{\delta_{z}\right\}$.

Proof. Let $z \in \Omega$. Assume that there exists $u \in \mathcal{P} \mathcal{S H}(\Omega) \cap C(\bar{\Omega})$ such that $u(z)=0$ and $u<0$ in $\bar{\Omega} \backslash\{z\}$. Then we get

$$
0=u(z) \leq \int_{\partial \Omega} u d \mu \leq 0 .
$$

Therefore supp $\mu \subset\{z\}$, so $\mathcal{J}_{z}^{c}=\left\{\delta_{z}\right\}$.

Proposition 2.6. Let $D_{j}$ be a bounded B-regular domain in $\mathbb{C}^{n_{j}}$, and let $D=D_{1} \times \cdots \times D_{s} \subset \mathbb{C}^{n}$, where $n=n_{1}+\cdots+n_{s}$. Fix $k \in\{1, \ldots, s-1\}$, $1 \leq j_{1}<\cdots<j_{k} \leq s, z_{j_{l}} \in \partial D_{j_{l}}$ for $l=1, \ldots, k$ and $z_{m} \in D_{m}$ for $m \notin\left\{j_{1}, \ldots, j_{k}\right\}$. Let $z=\left(z_{1}, \ldots, z_{j_{1}}, \ldots, z_{j_{k}}, \ldots, z_{s}\right) \in \partial D$. If $\mu \in \mathcal{J}_{z}^{c}$, then $\operatorname{supp} \mu \subset \bar{D}_{1} \times \cdots \times\left\{z_{j_{1}}\right\} \times \cdots \times\left\{z_{j_{k}}\right\} \times \cdots \times \bar{D}_{s}$.

If $z \in \partial D^{+}$, then $\mathcal{J}_{z}^{c}=\left\{\delta_{z}\right\}$. 
Proof. Let $z_{0} \in \partial D \backslash \partial D^{+}$, e.g. $z_{0}=\left(z_{1}, \ldots, w_{j_{1}}, \ldots, w_{j_{k}}, \ldots, z_{s}\right)$. where $z_{j} \in D_{j}, w_{j_{k}} \in \partial D_{j_{k}}$. Let $h_{j_{k}}$ be a strong plurisubharmonic barrier at $w_{j_{k}}$ for $D_{j_{k}}$. Define $u_{j_{k}}(z)=h_{j_{k}}\left(z_{j_{k}}\right)$, and let $\mu \in \mathcal{J}_{z_{0}}^{c}$. Then

$$
0=u_{j_{k}}(z) \leq \int_{\partial D} u_{j_{k}} d \mu
$$

which implies that

$$
\operatorname{supp} \mu \subset \bar{D}_{1} \times \cdots \times\left\{w_{j_{k}}\right\} \times \cdots \times \bar{D}_{s} .
$$

Hence,

$$
\begin{aligned}
\operatorname{supp} \mu & \subset \bigcap_{j_{k}} \bar{D}_{1} \times \cdots \times\left\{w_{j_{k}}\right\} \times \cdots \times \bar{D}_{s} \\
& =\bar{D}_{1} \times \cdots \times\left\{w_{j_{1}}\right\} \times \cdots \times\left\{w_{j_{k}}\right\} \times \cdots \times \bar{D}_{s} .
\end{aligned}
$$

To prove the second part of the proposition, we will show that for each $z \in$ $\partial D^{+}$there exists a strong plurisubharmonic barrier at $z$; then Proposition 2.5 will finish the proof. Let $\left(w_{1}, \ldots, w_{s}\right) \in \partial D^{+}$. Then for each $j$ there exists $\varphi_{j} \in \mathcal{P} \mathcal{S H}\left(D_{j}\right) \cap C\left(\bar{D}_{j}\right)$ such that $\varphi_{j}\left(w_{j}\right)=0$ and $\varphi_{j}<0$ in $\bar{D}_{j} \backslash\left\{w_{j}\right\}$. Now define

$$
u\left(z_{1}, \ldots, z_{s}\right)=\sum_{j=1}^{s} \varphi_{j}\left(z_{j}\right) .
$$

Then $u \in \mathcal{P S H}(D) \cap C(\bar{D}), u(w)=0$ and $u<0$ in $\bar{D} \backslash\{w\}$.

Let $\Omega \subseteq \mathbb{C}^{n}$ be a bounded hyperconvex domain and let $f: \partial \Omega \rightarrow \mathbb{R}$ be a continuous function. The Perron-Bremermann envelope is defined by

$$
\operatorname{PB}_{f}(z)=\sup \left\{w(z): w \in \mathcal{P} \mathcal{S H}(\Omega), \limsup _{\Omega \ni \zeta \rightarrow \xi} w(\zeta) \leq f(\xi) \forall \xi \in \partial \Omega\right\} .
$$

Hence $\mathrm{PB}_{f}$ is always plurisubharmonic, but not necessarily continuous. In [10] Walsh proved that if

$$
\liminf _{\Omega \ni z \rightarrow \xi} \operatorname{PB}_{f}(z)=\limsup _{\Omega \ni z \rightarrow \xi} \operatorname{PB}_{f}(z)=f(\xi)
$$

for every $\xi \in \partial \Omega$, then $\mathrm{PB}_{f} \in C(\bar{\Omega})$. We will refer to this result as Walsh's theorem.

\section{Proof of the equivalence (1)-(4) in Theorem A}

Lemma 3.1. Let $U$ be an open neighborhood of $\bar{D}$ defined in Section 2 and let $\pi: U \rightarrow \mathbb{C}^{n}$ be a proper holomorphic map. Let $\Omega_{\pi}:=\pi(D)$ and let $f: \partial \Omega_{\pi} \rightarrow \mathbb{R}$ be a continuous function. If there exists $u \in \mathcal{P S H}\left(\Omega_{\pi}\right) \cap$ $C\left(\bar{\Omega}_{\pi}\right)$ such that $\left.u\right|_{\partial \Omega_{\pi}}=f$, then $f$ is plurisubharmonic in the sense of Definition 2.2. 
Proof. Let $I_{k}$ be an increasing multi-index of length $k<s$, and $w_{j_{1}} \in$ $\partial D_{j_{1}}, \ldots, w_{j_{k}} \in \partial D_{j_{k}}$. Let $f_{w_{j_{1}}, \ldots, w_{j_{k}}}: D_{I_{k}} \rightarrow \mathbb{R} \cup\{-\infty\}$ be defined as in (2.1). We need to prove that this function is plurisubharmonic under the assumption that there exists $u \in \mathcal{P} \mathcal{S H}\left(\Omega_{\pi}\right) \cap C\left(\bar{\Omega}_{\pi}\right)$ such that $\left.u\right|_{\partial \Omega_{\pi}}=f$. Take a sequence $\left[\left(w_{j_{1}}^{m}, \ldots, w_{j_{k}}^{m}\right)\right]_{m=1}^{\infty}$ in $D_{j_{1}} \times \cdots \times D_{j_{k}}$ which converges to $\left(w_{j_{1}}, \ldots, w_{j_{k}}\right)$ as $m \rightarrow \infty$. Moreover, let $\left[u_{m}\right]$ be the sequence of real-valued functions on $D_{I_{k}}$ defined by

$$
u_{m}\left(z_{1}, \ldots, z_{s-k}\right)=u \circ \pi\left(z_{1}, \ldots, w_{j_{1}}^{m}, \ldots, w_{j_{k}}^{m}, \ldots, z_{s-k}\right) .
$$

This construction implies that $u_{m}$ is plurisubharmonic on $D_{I_{k}}$ and continuous up to the boundary. The sequence $\left[u_{m}\right]$ converges uniformly to $f_{w_{j_{1}}, \ldots, w_{j_{k}}}$ on $D_{I_{k}}$ as $m \rightarrow \infty$, and therefore $f$ is plurisubharmonic in the sense of Definition 2.2 .

Next we prove a characterization of those continuous boundary values which can be extended to continuous plurisubharmonic functions inside the domain.

Proposition 3.2. Let $D=D_{1} \times \cdots \times D_{s}$, where $D_{j}$ is a B-regular domain in $\mathbb{C}^{n_{j}}, j=1, \ldots s$, and let $f: \partial D \rightarrow \mathbb{R}$ be a continuous function. The following conditions are then equivalent:

(1) there exists $u \in \mathcal{P} \mathcal{S H}(D) \cap C(\bar{D})$ such that $\left.u\right|_{D}=f$,

(2) $f$ is plurisubharmonic in the sense of Definition $2.2\left(\right.$ with $\left.\pi=\mathrm{id}_{D}\right)$.

Proof. $(2) \Rightarrow(1)$ : If $z \in \partial D^{+}$, then $\mathcal{J}_{z}^{c}=\left\{\delta_{z}\right\}$ by Proposition 2.6. If $z \in \partial D \backslash \partial D^{+}$then there exist $k<s$ and $1 \leq j_{1}<\cdots<j_{k} \leq s$ such that $z_{j_{l}} \in \partial D_{j_{l}}$ for $l=1, \ldots, k$ and $z_{m} \in D_{m}$ for $m \notin\left\{j_{1}, \ldots, j_{k}\right\}$. If $\mu \in \mathcal{J}_{z}^{c}$, then

$$
\operatorname{supp} \mu \subset \bar{D}_{1} \times \cdots \times\left\{z_{j_{1}}\right\} \times \cdots \times\left\{z_{j_{k}}\right\} \times \cdots \times \bar{D}_{s},
$$

by Proposition 2.6. By our assumption $f$ is plurisubharmonic on $D_{1} \times \cdots \times$ $\left\{z_{j_{1}}\right\} \times \cdots \times\left\{z_{j_{k}}\right\} \times \cdots \times D_{s}$ and then by definition of Jensen measures we get

$$
f(z) \leq \int_{\bar{D}_{1} \times \cdots \times\left\{z_{j_{1}}\right\} \times \cdots \times\left\{z_{j_{k}}\right\} \times \cdots \times \bar{D}_{s}} f d \mu .
$$

Taking $\mu=\delta_{z}$ we find that for all $z \in \partial D$,

$$
f(z)=\inf \left\{\int_{\partial D} f d \mu: \mu \in \mathcal{J}_{z}^{c}\right\} .
$$

Then from Theorem 3.5 in [11] there exists $u \in \mathcal{P S H}(D) \cap C(\bar{D})$ such that $\left.u\right|_{D}=f$.

$(1) \Rightarrow(2)$ : Follows from Lemma 3.1 .

Lemma 3.3. Let $D$ be a bounded hyperconvex domain in $\mathbb{C}^{n}$, and let $U$ be an open neighborhood of $\bar{D}$. Let $\pi: U \rightarrow \mathbb{C}^{n}$ be a proper holomorphic 
map and let $\Omega_{\pi}=\pi(D)$. If $f: \partial \Omega_{\pi} \rightarrow \mathbb{R}$ is a continuous function such that $\mathrm{PB}_{f \circ \pi} \in \mathcal{P} \mathcal{S H}(D) \cap C(\bar{D})$ and $\mathrm{PB}_{f \circ \pi}=f \circ \pi$ on $\partial D$, then

$$
\mathrm{PB}_{f \circ \pi}=\mathrm{PB}_{f} \circ \pi \text {. }
$$

Furthermore, $\mathrm{PB}_{f \circ \pi}$ is pluriharmonic in $D$ if, and only if, $\mathrm{PB}_{f}$ is pluriharmonic on $\Omega_{\pi}$.

Proof. Define $g=f \circ \pi: \partial D \rightarrow \mathbb{R}$. By our assumption, $\mathrm{PB}_{g}$ is plurisubharmonic on $D$ and continuous on $\bar{D}$. Set

$$
\varphi(w)=\max \left\{\mathrm{PB}_{g}(z): z \in \pi^{-1}(w)\right\} .
$$

Then $\varphi \in \mathcal{P S H}\left(\Omega_{\pi}\right) \cap C\left(\Omega_{\pi}\right)$ (see [7]). We prove that $\left.\varphi\right|_{\partial \Omega_{\pi}}=f$. Let $\Omega_{\pi} \ni w_{j} \rightarrow w \in \partial \Omega_{\pi}$. Then there exist finitely many $z_{j}^{1}, \ldots, z_{j}^{k_{j}} \in \pi^{-1}\left(w_{j}\right)$. Take $z_{j}^{l_{j}}$ such that $\varphi\left(w_{j}\right)=\operatorname{PB}_{g}\left(z_{j}^{l_{j}}\right)$. Since $\pi$ is a proper map, we have $z_{j}^{l_{j}} \rightarrow z_{0} \in \partial D$ and then $\varphi\left(w_{j}\right)=\operatorname{PB}_{g}\left(z_{j}^{l_{j}}\right) \rightarrow \mathrm{PB}_{g}\left(z_{0}\right)=g\left(z_{0}\right)=f\left(\pi\left(z_{0}\right)\right)=$ $f\left(w_{0}\right)$.

Hence $\varphi \leq \operatorname{PB}_{f} \in \mathcal{P} \mathcal{S H}\left(\Omega_{\pi}\right) \cap C\left(\bar{\Omega}_{\pi}\right)$, by Walsh's theorem. Therefore $\mathrm{PB}_{f} \circ \pi \in \mathcal{P S H}(D)$ and $\left.\left(\mathrm{PB}_{f} \circ \pi\right)\right|_{\partial D}=g$. Thus, for $z \in \pi^{-1}(w)$ we get $\left(\mathrm{PB}_{f} \circ \pi\right)(z) \leq \mathrm{PB}_{g}(z)$ and so $\mathrm{PB}_{f}(w) \leq \varphi(w)$, which implies that $\varphi=\mathrm{PB}_{f}$. Therefore

$$
\mathrm{PB}_{f \circ \pi}=\mathrm{PB}_{f} \circ \pi
$$

since both functions are maximal with the same boundary values $g$.

Now we prove the second part of the lemma. From the first part it is clear that if $\mathrm{PB}_{f}$ is pluriharmonic on $\Omega_{\pi}$, then $\mathrm{PB}_{f \circ \pi}$ is pluriharmonic on $D$.

Now assume that $\mathrm{PB}_{g}$ is pluriharmonic on $D$. Note that $\mathrm{PB}_{g}=-\mathrm{PB}_{-g}$, since $\mathrm{PB}_{g}$ pluriharmonic on $D$ and continuous on $\bar{D}$. Hence by the first part of the proof,

$$
\begin{aligned}
\operatorname{PB}_{f}(w) & =\max \left\{\mathrm{PB}_{g}(z): z \in \pi^{-1}(w)\right\}=\max \left\{-\mathrm{PB}_{-g}(z): z \in \pi^{-1}(w)\right\} \\
& =-\min \left\{\mathrm{PB}_{-g}(z): z \in \pi^{-1}(w)\right\} .
\end{aligned}
$$

Similarly, $\mathrm{PB}_{-f}(w)=\max \left\{\mathrm{PB}_{-g}(z): z \in \pi^{-1}(w)\right\}$. Combining these two representations we obtain

$$
\begin{aligned}
0 \geq \mathrm{PB}_{f}+\mathrm{PB}_{-f}= & \max \left\{\mathrm{PB}_{-g}(z): z \in \pi^{-1}(w)\right\} \\
& -\min \left\{\mathrm{PB}_{-g}(z): z \in \pi^{-1}(w)\right\} \geq 0,
\end{aligned}
$$

so $\mathrm{PB}_{f}=-\mathrm{PB}_{-f}$, which means that $\mathrm{PB}_{f}$ is pluriharmonic.

We are now in a position to prove the first part of Theorem A.

Proof of Theorem A. (1) $\Rightarrow(2)$ : Follows immediately from Lemma 3.1.

$(3) \Rightarrow(1)$ : Obvious.

$(3) \Rightarrow(4)$ : Follows from Theorem 2.4 in [2]. 
$(4) \Rightarrow(2)$ : Let $I_{k}$ be an increasing multi-index $1 \leq j_{1}<\cdots<j_{k} \leq s$ of length $k<s$, and $z_{0} \in \Lambda^{I_{k}}$. Take any complex line $l$ through $z_{0}$ and $r>0$ such that $z_{0}+r \mathbb{E} \subset l \cap \Lambda^{I_{k}}$, where $\mathbb{E}$ is in $\mathbb{C}$. Since the Lebesgue measure $\lambda$ on the unit disc $\mathbb{E}$ is a Jensen measure at $z_{0}$, the measure $\mu_{\pi}(A)=\lambda\left(\pi^{-1}(A)\right)$, where $A \subset z_{0}+r \mathbb{E}$, is a Jensen measure at $\pi\left(z_{0}\right)$. By assumption,

$$
f\left(\pi\left(z_{0}\right)\right)=\int_{\pi\left(z_{0}+r \mathbb{E}\right)} f d \mu_{\pi}=\int_{z_{0}+r \mathbb{E}} f \circ \pi d \lambda,
$$

which implies that $f$ is harmonic on $\pi\left(z_{0}+r \mathbb{E}\right)$ and therefore pluriharmonic on $\partial \Omega_{\pi}$.

$(2) \Rightarrow(3):$ Let $g=f \circ \pi: \partial D \rightarrow \mathbb{R}$. Then $g$ is pluriharmonic on $\partial D$ and therefore Theorem 3.3 in [2] implies that $\mathrm{PB}_{g}$ is pluriharmonic on $D$, continuous on $\bar{D}$ and $\mathrm{PB}_{g}=g$ on $\partial D$. Therefore Lemma 3.3 finishes the proof.

4. The final part of Theorem A. We prove the following theorem.

Theorem 4.1. Assume that $\Omega \subseteq \mathbb{C}^{n}$ is a bounded hyperconvex domain having the approximation property and that $f: \partial \Omega \rightarrow \mathbb{R}$ is a continuous function. The following assertions are then equivalent:

(1) for every $\xi \in \partial \Omega$,

$$
\limsup _{\Omega \ni z \rightarrow \xi}\left(\mathrm{PB}_{f}+\mathrm{PB}_{-f}\right)(z)=0,
$$

(2) for every $z_{0} \in \partial \Omega$ and every $\mu \in \mathcal{J}_{z_{0}}$,

$$
f\left(z_{0}\right)=\int_{\partial \Omega} f d \mu
$$

(3) $\mathrm{PB}_{f}, \mathrm{~PB}_{-f} \in C(\bar{\Omega}) \cap \mathcal{P S H}(\Omega), \mathrm{PB}_{f}=f$ and $\mathrm{PB}_{-f}=-f$ on $\partial \Omega$.

Proof. $(3) \Rightarrow(1)$ : Obvious.

$(2) \Rightarrow(3)$ : Lemma 3.3 in [11] implies that there exist $u, v \in \mathcal{P S H}(\Omega) \cap$ $C(\bar{\Omega})$ such that

$$
\lim _{\Omega \ni z \rightarrow \zeta} u(z)=f(\zeta) \text { and } \lim _{\Omega \ni z \rightarrow \xi} v(z)=-f(\xi)
$$

for all $\zeta, \xi \in \partial \Omega$, hence

$$
\lim _{\Omega \ni z \rightarrow \zeta} \mathrm{PB}_{f}(z)=f(\zeta) \text { and } \lim _{\Omega \ni z \rightarrow \xi} \mathrm{PB}_{-f}(z)=-f(\xi),
$$

and by [10] we get $\mathrm{PB}_{f}, \mathrm{~PB}_{-f} \in C(\bar{\Omega}) \cap \mathcal{P} \mathcal{S H}(\Omega)$.

$(1) \Rightarrow(2)$ : First we will prove that assumption (4.1) implies that

$$
\limsup _{\Omega \ni z \rightarrow \zeta} \mathrm{PB}_{f}(z)=f(\zeta) \text { and } \limsup _{\Omega \ni z \rightarrow \xi} \mathrm{PB}_{-f}(z)=-f(\xi)
$$


for all $\zeta, \xi \in \partial \Omega$. Assume that this is not the case, for example there exists a $\xi \in \partial \Omega$ such that $\lim \sup _{z \rightarrow \xi} \mathrm{PB}_{f}(z)<f(\xi)$. Then

$$
\begin{aligned}
0 & =\limsup _{\Omega \ni z \rightarrow \xi}\left(\mathrm{PB}_{f}+\mathrm{PB}_{-f}\right)(z) \leq \limsup _{\Omega \ni z \rightarrow \xi} \mathrm{PB}_{f}(z)+\limsup _{\Omega \ni z \rightarrow \xi} \mathrm{PB}_{-f}(z) \\
& <f(\xi)-f(\xi)=0,
\end{aligned}
$$

a contradiction, hence limsup $\mathrm{PB}_{f}=f$ and limsup $\mathrm{PB}_{-f}=-f$ on $\partial \Omega$.

Fix $z_{0} \in \partial \Omega$ and take $\mu \in \mathcal{J}_{z_{0}}=\mathcal{J}_{z_{0}}^{c}$. Then $\operatorname{supp} \mu \subset \partial \Omega$ and

$$
f\left(z_{0}\right)=\mathrm{PB}_{f}^{*}\left(z_{0}\right) \leq \int_{\bar{\Omega}} \mathrm{PB}_{f}^{*} d \mu=\int_{\partial \Omega} \mathrm{PB}_{f}^{*} d \mu=\int_{\partial \Omega} f d \mu .
$$

Thus

$$
f\left(z_{0}\right) \leq \inf \left\{\int_{\partial \Omega} f d \mu: \mu \in \mathcal{J}_{z_{0}}\right\} .
$$

If $\mu=\delta_{z_{0}}$, then we obtain

$$
f\left(z_{0}\right)=\inf \left\{\int_{\partial \Omega} f d \mu: \mu \in \mathcal{J}_{z_{0}}\right\} .
$$

A similar formula can be obtained for $-f$ and therefore

$$
\begin{aligned}
\inf \left\{\int_{\partial \Omega}-f d \mu: \mu \in \mathcal{J}_{z_{0}}\right\} & =-f\left(z_{0}\right)=-\inf \left\{\int_{\partial \Omega} f d \mu: \mu \in \mathcal{J}_{z_{0}}\right\} \\
& =\sup \left\{\int_{\partial \Omega}-f d \mu: \mu \in \mathcal{J}_{z_{0}}\right\} .
\end{aligned}
$$

Thus, for every $z_{0} \in \partial \Omega$ and every $\mu \in \mathcal{J}_{z_{0}}$,

$$
f\left(z_{0}\right)=\int_{\partial \Omega} f d \mu
$$

and the proof is complete.

Proof of the final part of Theorem $A$. $(5) \Rightarrow(6)$ : If a bounded plurisubharmonic function $u$ on a bounded hyperconvex domain $\Omega$ has smallest maximal plurisubharmonic majorant identically zero, then $\lim \sup _{z \rightarrow \xi \in \partial \Omega} u(z)=0$.

$(3) \Rightarrow(5)$ : Obvious.

$(6) \Rightarrow(4)$ : Follows from Theorem 4.1.

5. Plurisubharmonicity in terms of analytic discs. Let $\mathbb{E}$ be the open unit disc in $\mathbb{C}$. Let $U$ be an open neighborhood of the closure of a bounded hyperconvex domain $D=D_{1} \times \cdots \times D_{n}$ in $\mathbb{C}^{n}$, and let $\pi: U \rightarrow \mathbb{C}^{n}$ be a proper holomorphic map. Set $\Omega_{\pi}:=\pi(D)$. Note that since each $D_{j}$ is a one-dimensional hyperconvex domain, it is also B-regular.

Proposition 5.1. Let $U$ be an open neighborhood of the closure of a bounded hyperconvex domain $D=D_{1} \times \cdots \times D_{n}$ in $\mathbb{C}^{n}, n \geq 2$, and let $\pi$ : 
$U \rightarrow \mathbb{C}^{n}$ be a proper holomorphic map. Let $\Omega_{\pi}:=\pi(D)$ and let $f: \partial \Omega_{\pi} \rightarrow \mathbb{R}$ be a continuous function. The following conditions are then equivalent:

(1) there exists $u \in \mathcal{P} \mathcal{S H}\left(\Omega_{\pi}\right) \cap C\left(\bar{\Omega}_{\pi}\right)$ such that $\left.u\right|_{\partial \Omega_{\pi}}=f$,

(2) $f$ is plurisubharmonic in the sense of Definition 2.2,

(3) $f$ is subharmonic on every analytic disc d embedded in $\partial \Omega_{\pi}$, i.e., $f \circ d$ is subharmonic on $\mathbb{E}$ for every injective, holomorphic function $d: \mathbb{E} \rightarrow \bar{\Omega}_{\pi}$ with $d(\mathbb{E}) \subseteq \partial \Omega_{\pi}$.

Proof. Since each $D_{j}$ is B-regular, the equivalence $(1) \Leftrightarrow(2)$ was proved in Lemma 3.3.

$(3) \Rightarrow(2)$ : Let $I_{k}$ be an increasing multi-index of length $k<n, w_{j_{1}} \in$ $\partial D_{j_{1}}, \ldots, w_{j_{k}} \in \partial D_{j_{k}}$. Let $f_{w_{j_{1}}, \ldots, w_{j_{k}}}: D_{I_{k}} \rightarrow \mathbb{R} \cup\{-\infty\}$ be defined as in (2.1). To prove that this function is plurisubharmonic, take $z_{0} \in D_{I_{k}}$. Let $\tilde{z}_{0}=\left(z_{1}, \ldots, w_{j_{1}}, \ldots, w_{j_{k}}, \ldots, z_{n}\right) \in \Lambda^{I_{k}}$, choose $X \in \mathbb{C}^{n-k}$ and let $\tilde{X}=\left(X_{1}, \ldots, X_{j_{l}}, \ldots, X_{n}\right)$, where $X_{j_{l}}=0$ for $l=1, \ldots, k$. Choose $r>0$ such that $\left\{\tilde{z}_{0}+\zeta r \tilde{X}: \zeta \in \mathbb{E}\right\} \subseteq \Lambda^{I_{k}}$. Let $d: \mathbb{E} \rightarrow \Lambda^{I_{k}}$ be an analytic disc embedded in $\Lambda^{I_{k}}$ defined by $d(\zeta)=\tilde{z}_{0}+\zeta r \tilde{X}$. Then $\pi \circ d$ is an analytic disc imbedded in $\Lambda_{\pi}^{I_{k}}$. Thus $f \circ \pi \circ d$ is subharmonic on $\mathbb{E}$ by assumption, hence $f_{w_{j_{1}}, \ldots, w_{j_{k}}}$ is plurisubharmonic on $\Lambda^{I_{k}}$.

$(2) \Rightarrow(3)$ : Let $d: \mathbb{E} \rightarrow \partial \Omega_{\pi}$. It is enough to show that there exists an increasing multi-index $I_{k}$ of length $k<n$ such that $d(\mathbb{E}) \subset \Lambda_{\pi}^{I_{k}}$. It is clear that $d(\mathbb{E}) \not \subset \pi\left((\partial \mathbb{E})^{n}\right)$. So there exist $z \in d(\mathbb{E})$ and an increasing multi-index $I_{k}$ of length $k<n$ such that $z \in \Lambda_{\pi}^{I_{k}}$.

We prove that $d(\mathbb{E}) \subset \Lambda_{\pi}^{I_{k}}$. Assume that it is not true. Then, since both sets are connected, there exist $\lambda_{1}, \lambda_{2} \in \mathbb{E}$ such that $d\left(\lambda_{1}\right) \in \Lambda_{\pi}^{I_{k}}$ and $d\left(\lambda_{1}\right) \in$ $\partial \Lambda_{\pi}^{I_{k}}$ (we can treat $\Lambda_{\pi}^{I_{k}}$ like a domain in $\mathbb{C}^{n-k}$ ). Therefore we can assume that there exists an analytic disc $\tilde{d}: \mathbb{E} \rightarrow \overline{\Lambda_{\pi}^{I_{k}}}$ such that $d\left(\lambda_{1}\right), d\left(\lambda_{2}\right) \in \tilde{d}(\mathbb{E})$. Let

$$
\begin{aligned}
\psi\left(z_{1}, \ldots, \hat{z}_{j_{1}}, \ldots, \hat{z}_{j_{k}}, \ldots, z_{n}\right) & \\
& \left.=\max \left(\phi_{1}\left(z_{1}\right), \ldots, \widehat{\phi_{j_{1}}\left(z_{j_{1}}\right)}, \ldots, \widehat{\phi_{j_{k}}\left(z_{j_{k}}\right.}\right), \ldots, \phi_{n}\left(z_{n}\right)\right)
\end{aligned}
$$

be an exhaustion function for $\Lambda^{I_{k}}$ treated like a subset of $\mathbb{C}^{n-k}$, where $\phi_{j}$ is an exhaustion function for $D_{j}$. We can consider the restriction $\pi: \mathbb{C}^{n-k} \supset$ $\Lambda^{I_{k}} \rightarrow \Lambda_{\pi}^{I_{k}} \subset \mathbb{C}^{n-k}$. Define

$$
v(w)=\max \left\{\psi(z): z \in \pi^{-1}(w)\right\} .
$$

Then $v$ is an exhaustion function for $\Lambda_{\pi}^{I_{k}}$. Define $h(\lambda)=v \circ \tilde{d}(\lambda)$. Then $h$ is a negative subharmonic function on $\mathbb{E}$. But on the other hand, $h\left(\lambda_{1}\right)<0$ and $h\left(\lambda_{2}\right)=0$, which is impossible.

Remark. Let $D=D_{1} \times \cdots \times D_{s}$, where each $D_{j}$ is a hyperconvex domain in $\mathbb{C}^{n_{j}}$ with $C^{1}$-boundary, $1 \leq j \leq s, s \geq 2$, and let $U$ be an open 
neighborhood of $D$. Let $\pi: U \rightarrow \mathbb{C}^{n}$ be a proper holomorphic map, where $n=n_{1}+\cdots+n_{s}$, and let $\Omega_{\pi}:=\pi(D)$. Following the idea from [2] one can show that the conclusion of Proposition 5.1 is also true for the $\Omega_{\pi}$ described above.

ThEOREM 5.2. Let $f \in \mathcal{C}(\partial \mathbb{E} \times \partial \mathbb{E})$, and let $d \sigma$ be the normalized Lebesgue measure on $\partial \mathbb{E}$. Then the Poisson integral of $f$ defined by

$$
P[f]\left(z_{1}, z_{2}\right)=\int_{\partial \mathbb{E} \times \partial \mathbb{E}} \frac{\left(1-\left|z_{1}\right|^{2}\right)\left(1-\left|z_{2}\right|^{2}\right)}{\left|w_{1}-z_{1}\right|^{2}\left|w_{2}-z_{2}\right|^{2}} f\left(w_{1}, w_{2}\right) d \sigma\left(w_{1}\right) d \sigma\left(w_{2}\right)
$$

is 2-harmonic (i.e. harmonic in each variable separately) on $\mathbb{E}^{2}$ and continuous on $\overline{\mathbb{E}^{2}}$. Furthermore, $P[f]$ is pluriharmonic in $\mathbb{E}^{2}$ if, and only if,

$$
\int_{\partial \mathbb{E} \times \partial \mathbb{E}} w_{1}^{k_{1}} \bar{w}_{2}^{k_{2}} f\left(w_{1}, w_{2}\right) d \sigma\left(w_{1}\right) d \sigma\left(w_{2}\right)=0
$$

for all $k_{1}, k_{2} \in \mathbb{N}$. Moreover, if $u$ is a 2 -harmonic function on $\mathbb{E}^{2}$, continuous on $\overline{\mathbb{E}^{2}}$, then $u=P[u]$.

Proof. See [8].

Using Rudin's result we obtain a similar result for $\partial \Omega_{\pi}$.

Proposition 5.3. Let $U$ be an open neighborhood of the closure of $\mathbb{E}^{2}$ in $\mathbb{C}^{2}$, and let $\pi: U \rightarrow \mathbb{C}^{2}$ be a proper holomorphic map. Let $\Omega_{\pi}:=\pi\left(\mathbb{E}^{2}\right)$, and let $f: \partial \Omega_{\pi} \rightarrow \mathbb{R}$ be a continuous function. The following are then equivalent:

(1) there exists a function $u$ which is pluriharmonic on $\Omega_{\pi}$, continuous on $\bar{\Omega}_{\pi}$ and $\left.u\right|_{\partial \Omega_{\pi}}=f$,

(2) $f$ is harmonic in the sense of Definition 2.2 and

$$
\int_{\partial \mathbb{E} \times \partial \mathbb{E}} w_{1}^{k_{1}} \bar{w}_{2}^{k_{2}} f\left(\pi\left(w_{1}, w_{2}\right)\right) d \sigma\left(w_{1}\right) d \sigma\left(w_{2}\right)=0
$$

for all $k_{1}, k_{2} \in \mathbb{N}$.

Proof. $(1) \Rightarrow(2)$ : Similarly to the proof of Lemma 3.1 one can show that $f$ is harmonic in the sense of Definition 2.2. By assumption it also follows that $\mathrm{PB}_{f}$ is pluriharmonic on $\Omega_{\pi}$. Therefore by Lemma 3.3, $\mathrm{PB}_{f \circ \pi}=\mathrm{PB}_{f} \circ \pi$ and $\mathrm{PB}_{f} \circ \pi$ is pluriharmonic on $\mathbb{E}^{2}$, continuous on $\overline{\mathbb{E}^{2}}$, and $\mathrm{PB}_{f} \circ \pi=f \circ \pi$ on $\partial \mathbb{E}^{2}$. Then $\mathrm{PB}_{f \circ \pi}=P[f \circ \pi]$ is the Poisson integral of $f \circ \pi$, so (5.1) holds by Theorem 5.2 .

$(2) \Rightarrow(1)$ : From (2) it follows that (5.1) holds for all $k_{1}, k_{2} \in \mathbb{N}$. By Theorem 5.2, $P[f \circ \pi]$ is pluriharmonic on $\mathbb{E}^{2}$. Since $f$ is harmonic in the sense of Definition 2.2, the function $P[f \circ \pi]=\mathrm{PB}_{f \circ \pi}$ is pluriharmonic on $\mathbb{E}^{2}$, continuous on $\partial \mathbb{E}^{2}$, and $\mathrm{PB}_{f \circ \pi}=f \circ \pi$ on $\partial \mathbb{E}^{2}$. Therefore by Lemma 3.3, $\mathrm{PB}_{f}$ is pluriharmonic on $\Omega_{\pi}$, and the proof is complete. 
Acknowledgements. The author would like to thank Per Åhag and Urban Cegrell for many valuable comments on and suggestions for this manuscript. He would also like to thank the referee for his valuable suggestions which helped to improve both the statements and proofs. This research was in part done during the author's visit to Umeå University, Sweden, in 2008. He would like to thank the members of the Department of Mathematics and Mathematical Statistics for their kind hospitality.

The author was partially supported by ministerial grant number N N201 367933.

\section{References}

[1] P. Åhag and R. Czyż, The connection between the Cegrell classes and compliant functions, Math. Scand. 99 (2006), 87-98.

[2] - - - Continuous pluriharmonic boundary values, Ann. Polon. Math. 91 (2007), 99-117.

[3] M. Carlehed, U. Cegrell and F. Wikström, Jensen measures, hyperconvexity and boundary behaviour of the pluricomplex Green function, Ann. Polon. Math. 71 (1999), 87-103.

[4] J. L. Doob, Classical Potential Theory and its Probabilistic Counterpart, Classics in Math., Springer, 2001.

[5] A. Edigarian and W. Zwonek, Geometry of the symmetrized polydisc, Arch. Math. (Basel) 84 (2005), 364-374.

[6] F. Forstnerič, Proper holomorphic mappings: a survey, in: Several Complex Variables (Stockholm, 1987/1988), Math. Notes 38, Princeton Univ. Press, Princeton, NJ, 1993, 297-363.

[7] M. Klimek, On the invariance of the L-regularity under holomorphic mappings, Zeszyty Nauk. Uniw. Jagielloń. Prace Mat. 23 (1982), 27-38.

[8] W. Rudin, Function Theory in Polydiscs, Benjamin, 1969.

[9] N. Sibony, Une classe de domaines pseudoconvexes, Duke Math. J. 55 (1987), 299319.

[10] J. B. Walsh, Continuity of envelopes of plurisubharmonic functions, J. Math. Mech. 18 (1968), 143-148.

[11] F. Wikström, Jensen measures and boundary values of plurisubharmonic functions, Ark. Mat. 39 (2001), 181-200.

Institute of Mathemathics

Jagiellonian University

Łojasiewicza 6

30-348 Kraków, Poland

E-mail: Rafal.Czyz@im.uj.edu.pl

Received 24.9.2008

and in final form 3.12.2008 